Torreón Universitario

www.faremcarazo.unan.edu.ni / ISSN 2410-5708 / e-ISSN 2313-7215

Año 8 | Núm. 23 | pág. 84 - pág. 101 | octubre 2019 - enero 2020
Atribución-NoComercial-SinDerivar 4.0 Internacional

\title{
Análisis de requerimiento y desarrollo de sistema de información gerencial de las variedades disponibles de granos básicos georreferenciadas, en el Departamento de Carazo (SIGITEC), en el periodo 2007-20I5
}

Ing. Álvaro Antonio Mejía Quiroz

Docente investigador UNAN-Managua, FAREM-Carazo alvaro.mejia.quiroz@gmail.com

Fecha de recepción: 02 de diciembre, 2019 / Fecha de aceptación: 28 de enero, 2020 https://doi.org/10.5377/torreon.v8i23.9535

Palabras clave: variedades de granos básicos, sistemas de información, parámetros biofísicos, agricultores, datos geográficos

\section{RESUMEN}

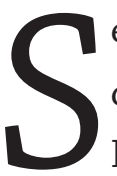

e presenta la creación de un sistema de información gerencial ${ }^{1}$ de las variedades disponibles de granos básicos georreferenciadas en Carazo liberadas por el Instituto Nicaragüense de Tecnología Agropecuaria (INTA), entre los años 2007 y 2015, que contribuye en gran medida a la gestión de información de estas. Desarrollado por la Unidad de Investigación y Desarrollo Tecnológico de la Facultad Regional Multidisciplinaria de Carazo (FAREM-Carazo), con apoyo de los Fondos para Investigación (FPI) de la UNANManagua; que permite ingresar información de las características, ubicación geográfica y análisis de idoneidad de las parcelas con respecto a las variedades de granos básicos. La investigación dio

1. Investigación: Sistema de información gerencial delas variedades disponibles de granos básicos georreferenciadas, en el Departamento de Carazo (SIGITEC), en el periodo 2007-2015, financiada por los fondos FPI de la UNANManagua, de la Maestría en Métodos de Investigación Científica MEDINV Segunda Cohorte. Línea de Investigación: Tecnologías, Innovación y Energía Renovable. Participante: Álvaro Antonio Mejía Quiroz, 2007-2019. 
como resultados: el análisis de los requisitos del sistema, las alternativas para la digitalización de los mapas de temperatura, precipitación, altitud y tipo de suelos del departamento de Carazo, también la disposición de los mapas, así como la elección de una alternativa de tecnología de análisis, diseño, desarrollo e implementación con componentes sólidamente estructurados. Este producto permite la gestión eficiente de las variedades y el análisis en tiempo real de la condiciónes biofísicas de las parcelas para la determinación de cuales variedades de granos básicos son adaptables a dichas, haciendo uso del Lenguaje de Programación C\# y la plataforma ArcGis Online para el manejo y administración de mapas de manera automática.

\section{INTRODUCCIÓN}

La agricultura es uno de los principales rubros, por no decir el más importante, que fortalece la economía de Nicaragua, según El Banco Mundial (2019) esta creció un 4.7 y 4.5 por ciento en 2016 y 2017, respectivamente, pero debido a los disturbios sociales y políticos que el país experimentó en abril de 2018, la economía se contrajo un 3.8 por ciento en 2018 y se contraería a -5 por ciento en 2019 , según los últimos pronósticos.

Esta situación dispone un escenario para Nicaragua, como una condición llena de oportunidades para establecer propuestas que impacten de manera contundente y transcendental en pro de la agricultura, el medio ambiente y la economía nicaragüense, haciendo uso pertinentemente de las tecnologías de la comunicación y la información (TIC) en proyectos que se ejecuten desde la academia en pro de la sociedad.

En la estrategia del Banco Interamericano de Desarrollo (2012), se plantean tres sectores en las áreas de acción transversal: (i) reducción a la vulnerabilidad, adaptación y mitigación al cambio climático, (ii) género y (iii) diversidad. Tomando en cuenta el primer sector, es necesario reconocer que las tecnologías de variedades de granos básicos se adaptan a condiciones biofísicas muy diversas y documentadas, pero el INTA no tiene un sistema abierto a la población que le permita sistematizar todas las variedades de granos básicos (arroz, maíz, frijol y sorgo) y darles a los productores una herramienta innovadora que agilice los procesos de adquisición de la semillas o el análisis en tiempo real de sus parcelas, esto no resta el gran trabajo que en cada delegación realizan los técnicos con los agricultores en la comunidades que atienden.

Durante la última década, la Tecnología de la Información y la Comunicación (TIC), se ha convertido en una solución para mejorar la producción agrícola. Esta tendencia puede reflejarse en las múltiples aplicaciones de software que brindan servicios para disminuir los efectos causados por enfermedades y plagas en los cultivos, o en el monitoreo en tiempo real de las condiciones climáticas y los parámetros de calidad del agua utilizados para la producción. Las actividades de gestión, la toma de decisiones y la gestión de eventos complejos son piedras angulares asumidas por las soluciones TIC aplicadas a la agricultura. 
Angelov, Iglesias, \& Corrales, (2017) y Corrales, Angelov, \& Iglesias, (2018), han sistematizado conferencias internacionales en Colombia donde se analizaron los problemas emergentes y las nuevas soluciones TIC para abordar los efectos del cambio climático y la variabilidad climática en el sector, proponiendo estrategias y directrices comunes para incorporar la gestión de riesgos y la adaptación al cambio climático. Temas como la agricultura inteligente, los sistemas de predicción de enfermedades o plagas, la eficiencia del agua, el clima y sus efectos en la producción de cultivos, el modelado de la cubierta terrestre para los sistemas forestales y de cultivos, la teledetección para la producción y gestión de cultivos, la integración de datos meteorológicos para la agricultura fueron cubiertos por los autores. La conferencia fue organizada por la Red Interinstitucional para el Cambio Climático y la Seguridad Alimentaria de Colombia-RICCLISA, COLCIENCIAS, Universidad del Cauca, Colombia (Grupo de Ingeniería Telemática) y la Universidad Carlos III de Madrid, España (grupo de investigación CAOS), y patrocinado técnicamente por Spring.

Los agricultores e instituciones tanto públicas como privadas relacionadas con el tema del agro, conocen muy poco sobre los tipos de tecnologías innovadoras que pueden usarse en la agricultura, esto ocasiona un desaprovechamiento de éstas mismas, a esto le sumamos que aún muchos productores utilizan prácticas agrícolas no amigables con el medio ambiente, que afectan directamente al clima y la degradación acelerada de los suelos.

Otro aspecto a tomar en cuenta, es el no aprovechamiento de manera oportuna de la base de conocimientos que se genera en las Universidades Públicas del país, a pesar de esta situación se están haciendo vinculaciones y presentación de avances en el tema en Nicaragua. El Instituto Nicaragüense de Tecnología Agropecuaria (INTA) está promoviendo el Premio Nacional a la Innovación para agricultores, asociaciones de agricultores, docentes y estudiantes investigadores, espacio que puede ser aprovechado para dar a conocer el avance de la propuesta de estudio del presente trabajo de investigación.

En Nicaragua existe una considerable cantidad de tecnologías agropecuarias. El Instituto Nicaragüense de Tecnología Agropecuaria, (2015) , menciona que "en el país existen 284 tecnologías de las cuales 231 han sido generadas por el INTA y 53 por las diferentes universidades del sector agropecuario". En el mismo catálogo, de acuerdo a estudios hechos en el campo, existen "117 tecnologías aplicables para la producción del país" y de estas 117 tecnologías "68 son variedades de semillas, 21 de bio-insumos, 9 implementos agrícolas, 11 sistemas de riegos y 7 de pos cosecha y almacenamiento".

El Instituto Nicaragüense de Tecnología Agropecuaria, (2019), en la dirección web http://www.inta.gob.ni/agricultura-inteligente, presenta todas las recomendaciones necesarias para los cultivos de granos básicos, hortalizas y legumbres, raíces y tubérculos, ganadería, 
industriales y exportación, musáceas, diversos y exportación, frutales y cucurbitáceas, por cada uno de los municipios del país, pero no lo hace de manera dinámica ni automática y presenta las condiciones óptimas para cada una de los rubros mencionados para las áreas específicas en el país.

Con el desarrollo de la primera etapa de esta investigación, se tiene como resultado la creación de un producto novedoso siendo este uno más de los que se han desarrollado por la Unidad de Investigación y Desarrollo Tecnológico(UIDT) en los últimos años, en específico con la línea de investigación que pertenece a la categoría de los Sistemas de Información para la Toma de Decisiones, particularmente en el ámbito de la aplicación de Tecnologías de Variedades en Granos Básicos (frijol, arroz, sorgo y maíz), añadiendo un componente muy importante como lo son los datos geográficos, que según Pérez Navarro, (2011), "son la representación digital de entidades, objetos o fenómenos que ocurren sobre la superficie de la Tierra o cerca de ésta." (Pág. 143).

En este sentido, la UNAN-Managua FAREM-Carazo fortalece las funciones de investigación y extensión con los agricultores y el Instituto INTA del departamento de Carazo. En este marco, el Gobierno de Reconciliación y Unidad Nacional (2017), en los ejes del Programa Nacional de Desarrollo Humano (PNDH), menciona que "las características de esta intervención se definen por una asistencia que integra a investigadores, extensionistas y productores con trabajo directo en fincas, con reconocimiento tanto de las potencialidades de la zona como de la cultura local de las familias rurales", esto advierte que las instituciones educativas están en la obligación de dar soluciones reales a las problemáticas del país en donde se pueda incidir positivamente.

\section{METODOLOGÍA}

Para el estudio se aplicaron diferentes metodologías que ayudaron en las distintas fases del proceso de investigación, desde las entrevistas a especialistas y agricultores para obtener la información que una vez procesada dio lugar a la definición de los requerimientos y a los alcances y limitantes del sistema, hasta el uso de metodologías propias del análisis, diseño e implementación del sistema para generar un producto fiable y que cumpla con los requerimientos que la metodología de la Programación Orientada a Objetos y el uso de los Sistemas de Información Geográfica, que según (Corrales et al., 2018), se define como, "las acciones organizadas con que las personas miden aspectos de fenómenos y procesos geográficos para enfatizar cuestiones espaciales, entidades y relaciones. Cabe mencionar que operan bajo estas representaciones, descubren nuevas relaciones mediante la integración de diferentes fuentes y transforman estas representaciones." 
El proceso para el desarrollo del sistema consistió en cuatro etapas generales:

1. La primera se basó en la determinación de los términos de referencia para poder determinar cada uno de los elementos necesarios para las siguientes dos etapa, y las actividades que se llevaron a cabo fueron las siguientes: entrevista grupal con docentes de la Facultad de Recursos Naturales (FARENA) de la Universidad Nacional Agraria (UNA), entrevista grupal con el delegado departamental del INTA y los 8 técnicos que trabajan en el departamento de Carazo y un grupo focal con un grupo de agricultores de la comunidad El Abra, del municipio de La Conquista.

2. La segunda consistió en el desarrollo de las bases de datos georreferenciadas de los municipios de Carazo que para su implementación en el sistema de información, esta se ejecutó con la digitalización de los mapas en el programa ArcGis 10.3 y luego el alojamiento en un servicio web de ArcGis Online. Los mapas temáticos que se alojaron en esta plataforma son: precipitación, elevación, temperatura y tipos de suelos.

3. La tercera etapa consistió en la implementación de la base de datos relacional de las tecnologías de granos básicos, datos que fueron obtenidos en la primera etapa, en el departamento de Carazo utilizando Microsoft SQL Server, Visual C\# y Entity Framework que según MicroSoft, (2019), permite a "los desarrolladores trabajar con datos en forma de objetos y propiedades específicos del dominio como clientes y direcciones de clientes sin tener que preocuparse de las tablas y columnas de base de datos subyacentes donde se almacenan estos datos", siendo esta combinación una herramienta de programación, siempre tomando en consideración los principios y estándares de web-forms, donde se utilizó el Lenguaje de Modelo Unificado (UML), basado en el Paradigma de la Programación Orientada a Objetos (POO) que centra la importancia de los procesos en el adecuado diseño de las estructuras de datos y por otra parte promueve la reutilización efectiva de software y facilita la escalabilidad del software en función de nuevas demandas del contexto.

4. La cuarta etapa consistió en la comunicación de los mapas alojados en ArcGis Online, con el Lenguaje de programación C\# y la vinculación con el gestor de bases de datos Microsoft SQL Server utilizando JavaScript, para esto se configuró un servicio web que permitió consumir en tiempo real la información de mapas alojadas.

Se utilizó Microsoft Project 2016 para la elaboración de los cronogramas de actividades realizadas, tanto en la fase de recopilación de la información como en la etapa del análisis, diseño y programación del sistema. 
Se utilizó la herramienta ArgoUML para la elaboración de los distintos diagramas que el paradigma UML utiliza para representar gráficamente los distintos procesos que la aplicación realiza durante su funcionamiento. Para el diseño de interfaces y el almacenamiento de la información se utilizó el entorno de desarrollo Visual Studio 2017 y 2019 en su versión final, utilizando los controles Telerik, y el Gestor de Base de Datos SQL Server 2018.

\section{RESULTADOS}

Uno de los resultados del estudio fue, la valoración con respecto a la factibilidad técnica para el desarrollo del sistema en cuanto a uno de los aspectos de los requisitos, tomando en cuenta el plazo para el desarrollo, el nivel de conocimiento en las plataformas de desarrollo propuestas, el análisis de la aplicación y la inversión en equipos para el desarrollo y alojamiento del sistema se tomaron en cuenta los siguientes criterios:

Tabla 1: Softwares que se tomaron en cuenta para la factibilidad técnica.

\begin{tabular}{|l|l|}
\hline \multicolumn{2}{|c|}{ SOFTWARE PARA EL DESARROLLO } \\
\hline IDE de Desarrollo & Visual Studio Community 2017 y 2019 \\
\hline Diseño Gráfico & Gimp 2.8 \\
\hline Diagramación UML & ArgoUML \\
\hline Lenguaje de Programación & C\# \\
\hline Lenguaje del lado del Cliente & JavaScript \\
\hline Ofimática & $\begin{array}{l}\text { Microsoft Office 2016 (ya se tenía } \\
\text { licencia) }\end{array}$ \\
\hline Entorno para publicación de la Web & IIS Express \\
\hline Plataforma de Alojamiento de Mapas & $\begin{array}{l}\text { ArcGis Online (con versión gratuita de } \\
50 \text { créditos mensuales) }\end{array}$ \\
\hline
\end{tabular}

Las entrevistas que se aplicaron tanto a docentes de la UNA como técnicos del INTA Carazo nos dieron los siguientes resultados, para establecer la siguiente información sobre los mapas temáticos y las características de las variedades de granos básicos.

En cuanto a la información de los mapas temáticos era necesario digitalizar: precipitación, elevación, temperatura y tipos de suelos, cabe recalcar que la información del pH del suelo y la humedad son necesarias pero no se cuenta con mapas, por esta razón estos parámetros se introducen manualmente. 


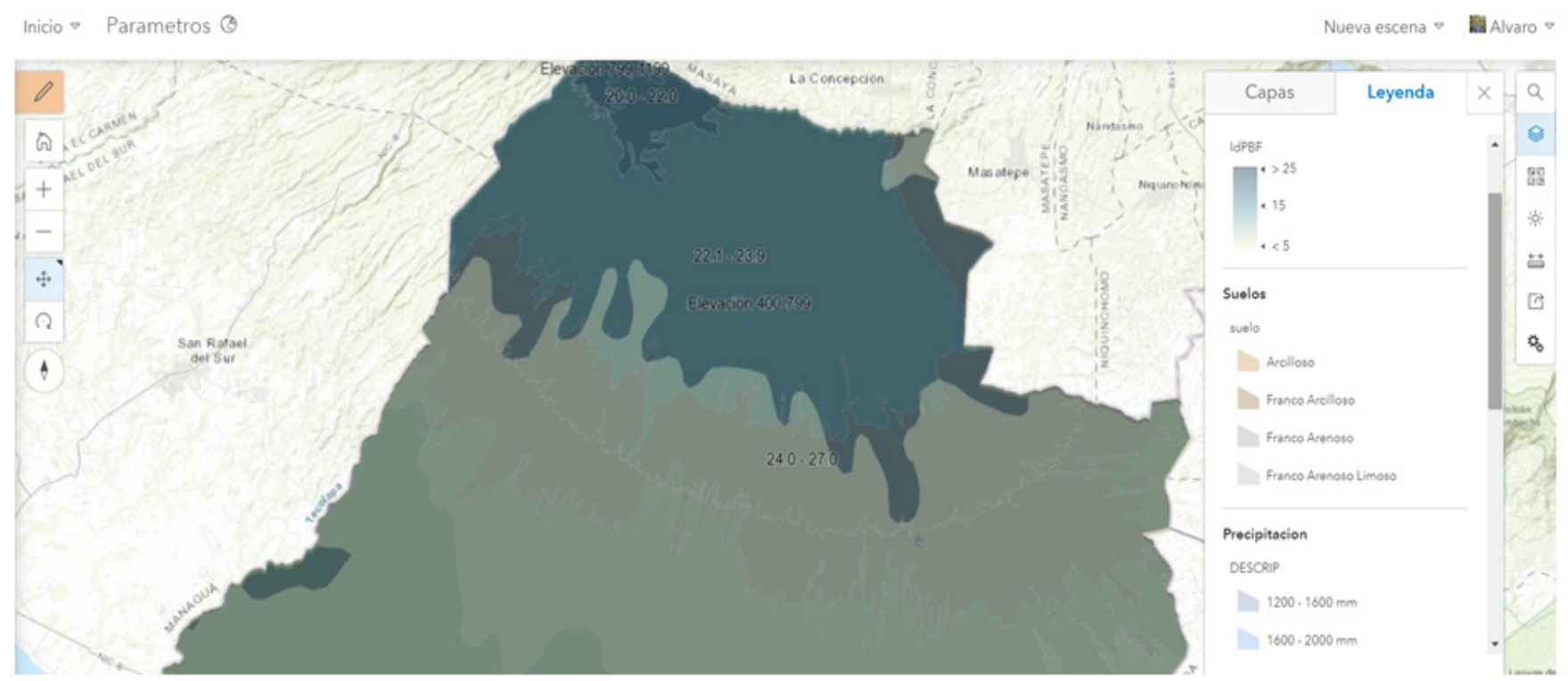

Figura 1: Mapas temáticos alojados en AcrGis Online.

$\begin{array}{lcc}\begin{array}{l}\text { Suelos } \\ \text { suelo }\end{array} & \begin{array}{c}\text { Precipitacion } \\ \text { Descilloso }\end{array} & \begin{array}{c}\text { Temperatura } \\ \text { DESCRIP }\end{array} \\ \text { Franco Arcilloso } & 1200 \cdot 1600 \mathrm{~mm} & 27.0 \cdot 22.0 \\ \text { Franco Arenoso } & 1600-2000 \mathrm{~mm} & 22.1 \cdot 23.9 \\ \text { Franco Arenoso Limoso } & 800 \cdot 1200 \mathrm{~mm} & 24.0 \cdot 27.0\end{array}$

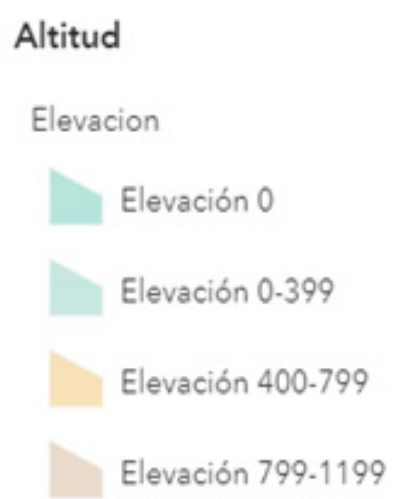

Figura 2: Valores para parametrizar cada elemento biofísico para los mapas.

Para los tipos de suelos en Carazo se obtuvo los tipos: arcillosos, franco arcilloso, franco arenoso, franco arenoso limoso. Para la precipitación se tiene tres categorías historias con valores de mm: 800-1200, 1200-1600, 1600-2000. Para la temperatura se tienen rango de grados Celsius: $20.00^{\circ}-22.00^{\circ}, 22.1^{\circ}-23.9^{\circ}, 24.0^{\circ}-27.0^{\circ}$ y mayor que $27^{\circ}$ y por último los valores de elevación estando en rangos de: 0 msnm, 0-399mnsm, 400-799 msnm y 799-1199 msnm.

La información que resultó necesaria para las variedades de granos básicos con base en las entrevistas para cada variedad es la siguiente: 
Tabla 2: Información necesaria para la base de datos relacional.

\begin{tabular}{|c|c|}
\hline Cuantitativas & Cualitativas \\
\hline Fecha & País \\
\hline Año de liberación de la variedad & Departamento \\
\hline Tamaño de la planta (centímetros) & Municipio \\
\hline Altura de inserción mazorca & INIA \\
\hline Altura inserción vaina & Investigador \\
\hline Longitud de ecxerción de la panoja & Email \\
\hline Longitud de ecxerción de la panícula & Cédula \\
\hline Rendimiento potencial (Kg/Ha) & Rubro \\
\hline Tiempo de cocción & Nombre de la Variedad \\
\hline Área cultivada (ha) & Sinónimos de la variedad \\
\hline Volumen de producción (Ton) & Instituciones responsables de la liberación \\
\hline Rendimiento promedio (Ton/Ha) & Fotos de las plantas \\
\hline Rendimiento promedio materia verde (Ton/ & Nombre de los progenitores \\
\hline $\mathrm{Ha})$ & Origen de los Progenitores \\
\hline Rendimiento promedio materia seca (Ton/Ha) & Características Genéticas \\
\hline Rendimiento promedio espiga (Ton/Ha) & Polinización Libre \\
\hline Rendimiento promedio de Fibra (Ton/Ha) & Hibrido simple \\
\hline Estimación del grado de adopción (\%) & Hibrido doble \\
\hline Latitud y longitud (coordenadas proyectadas & Hibrido triple \\
\hline en UTM) & Otras \\
\hline Cantidad de semilla genética $(\mathrm{Kg})$ & Hábito de crecimiento \\
\hline Cantidad semilla básica (Kg) & Color de grano \\
\hline Cantidad semilla registrada (Kg) & Habilidad de macoyamiento \\
\hline Cantidad de semilla certificada (Kg) & Adaptación a suelos con limitantes \\
\hline \multirow[t]{12}{*}{ Cantidad de semilla de otra categoría (Kg) } & Tolerancia a patógenos \\
\hline & Épocas del año de siembra \\
\hline & Calidad de las proteínas \\
\hline & Biofortificada \\
\hline & Empresa que produce la variedad \\
\hline & Distribuidor \\
\hline & Adaptabilidad a rangos de altitud \\
\hline & Adaptabilidad a tipos de suelos \\
\hline & Adaptabilidad a precipitación pluvial \\
\hline & Adaptabilidad a rangos de temperatura \\
\hline & Adaptabilidad a porcentajes de humedad \\
\hline & Adaptabilidad al pH del suelo \\
\hline
\end{tabular}


Con base en esta información se pudo tener la base de datos:

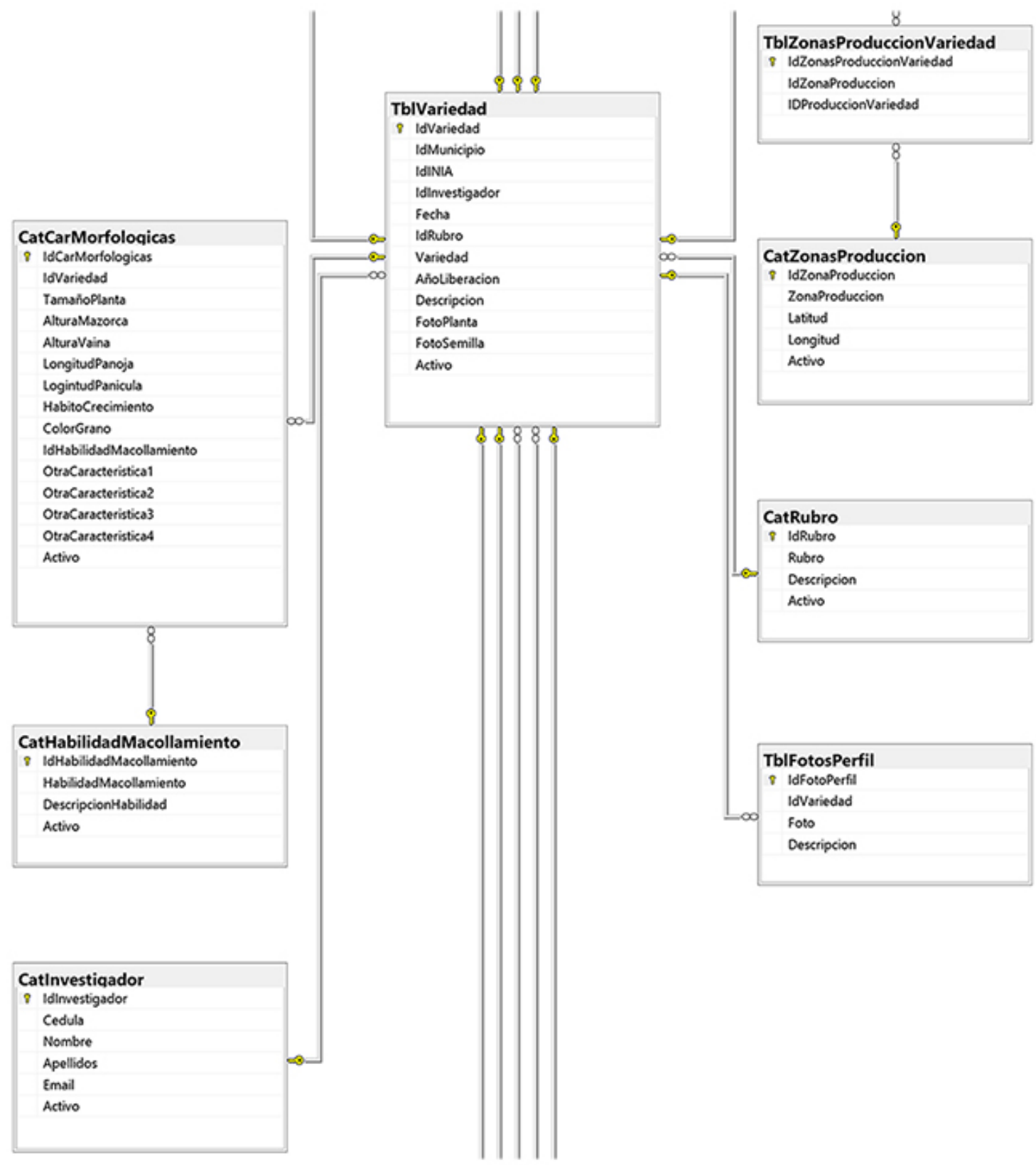

Figura 3: Parte de la base de datos relacional normalizada que se obtuvo del análisis.

Una vez teniendo cada uno de los componentes conceptuales del sistema, se procedió a la creación de los módulos, que permitió el funcionamiento en ambiente Web. Seguidamente, se muestran las interfaces principales de la aplicación que son parte del resultado del estudio. 


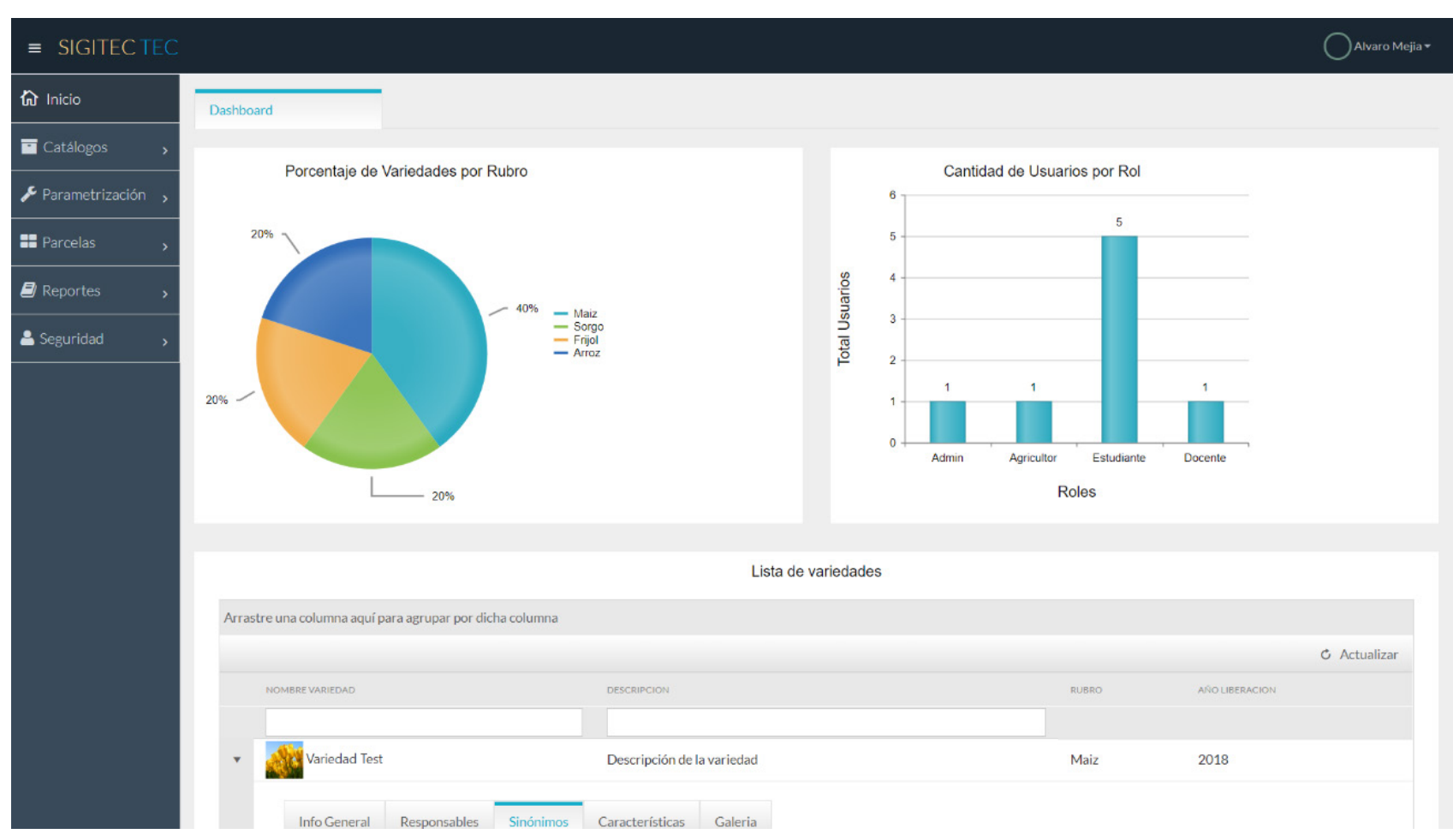

Figura 4: Administración principal del Sistema.

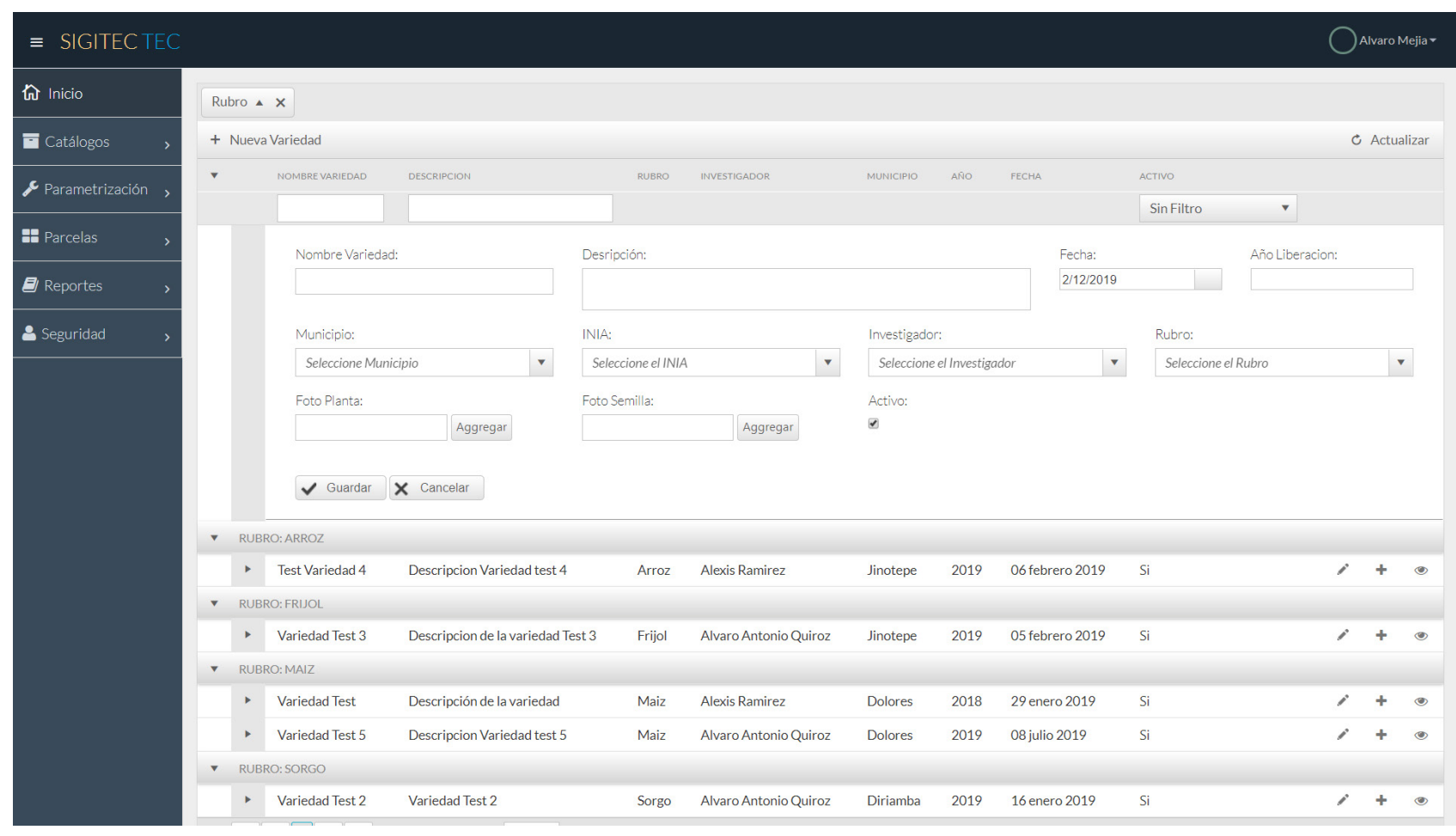

Figura 5: Administración de las Variedades. 


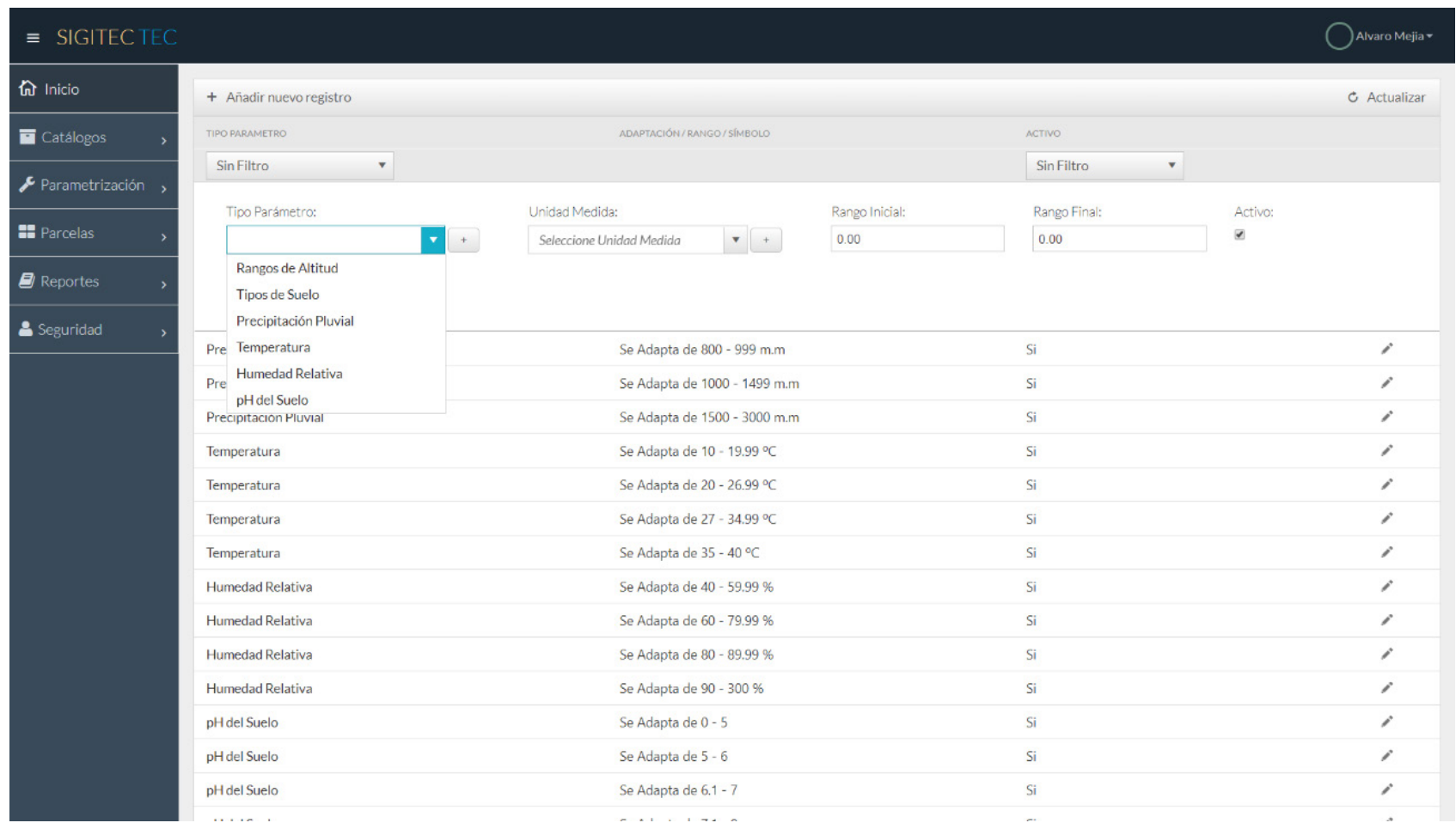

Figura 6: Administración de cada parámetro que pueda existir para adaptabilidad de las variedades.

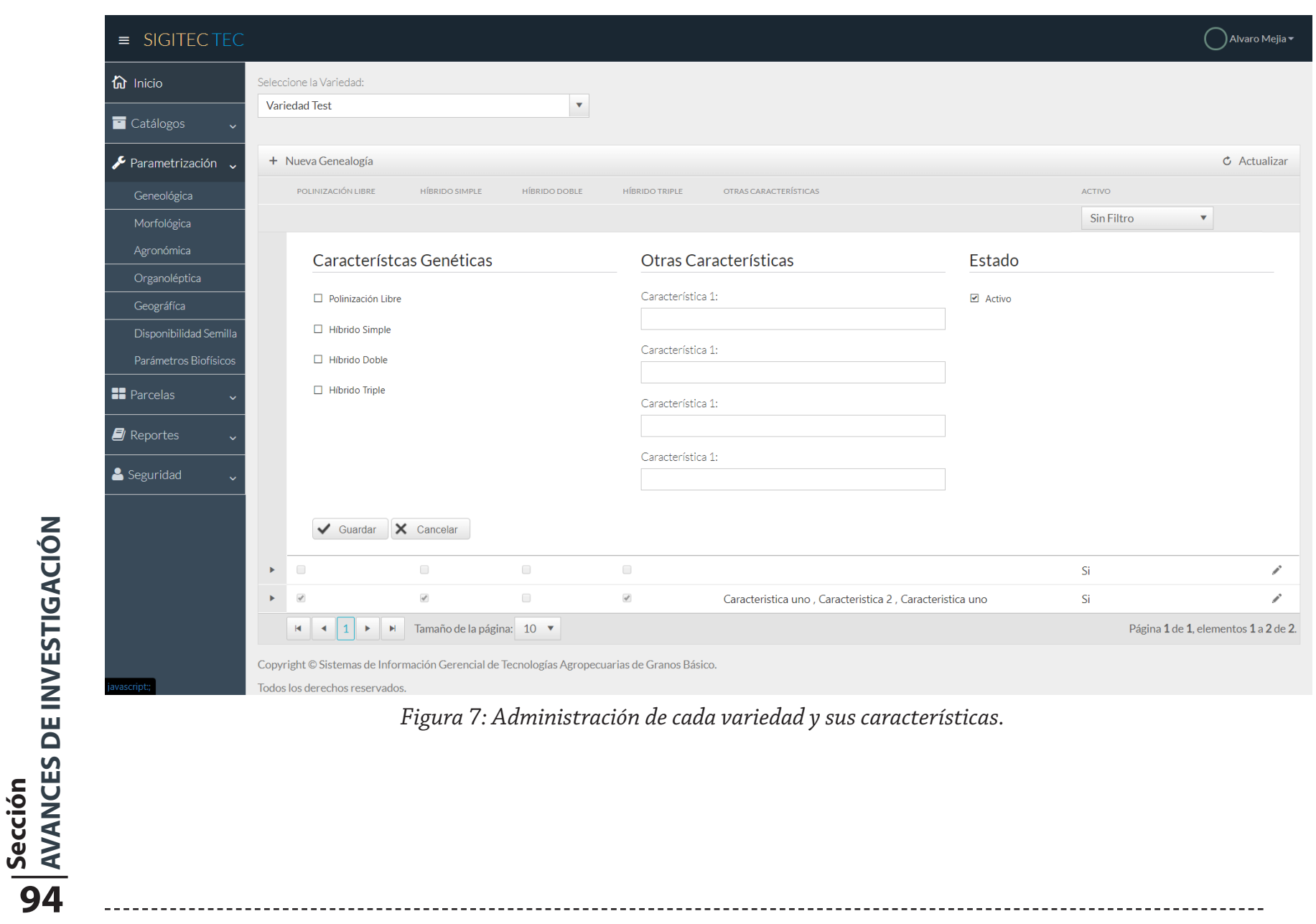




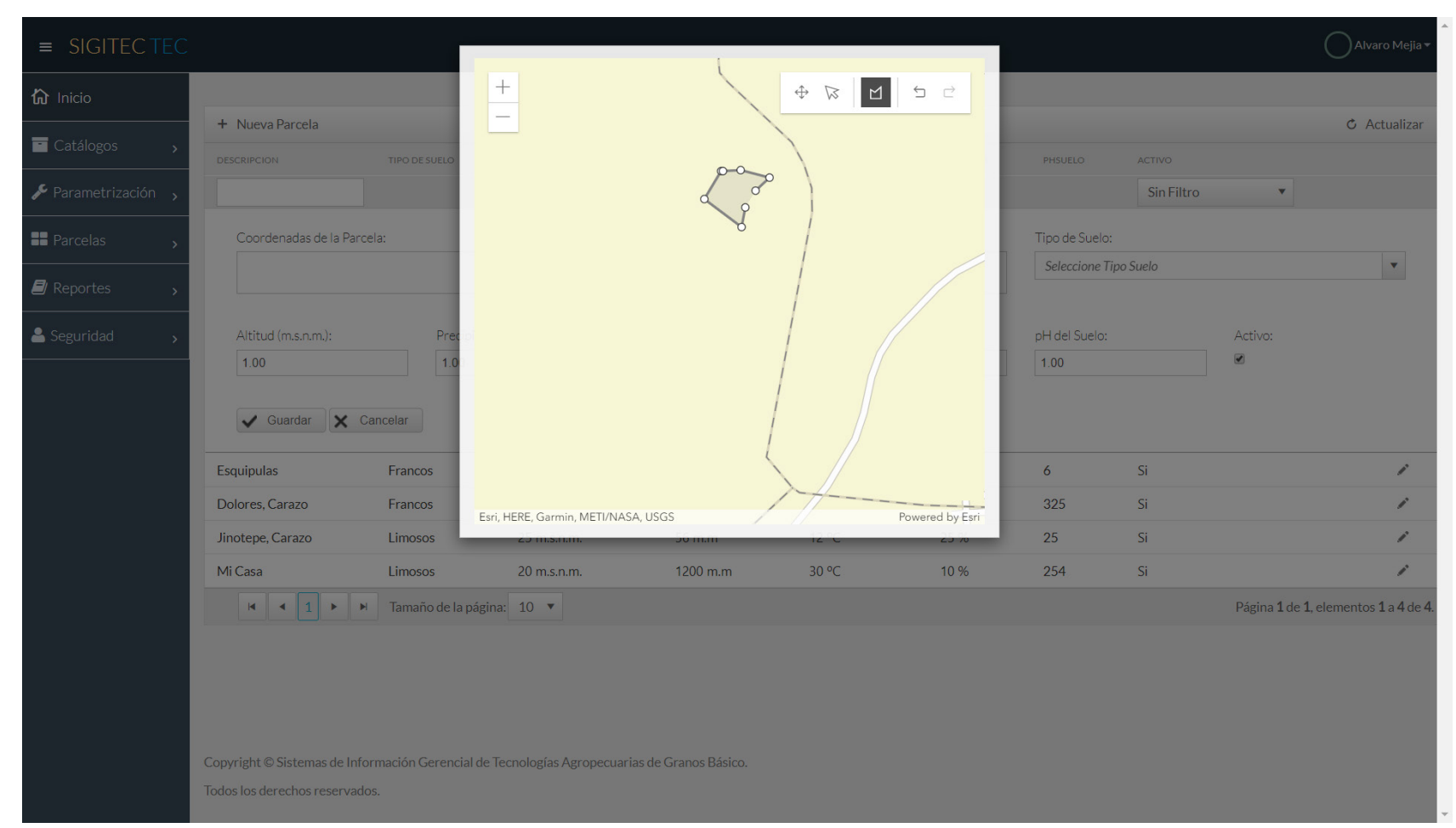

Figura 8: Administración de cada parcela para su análisis correspondiente.

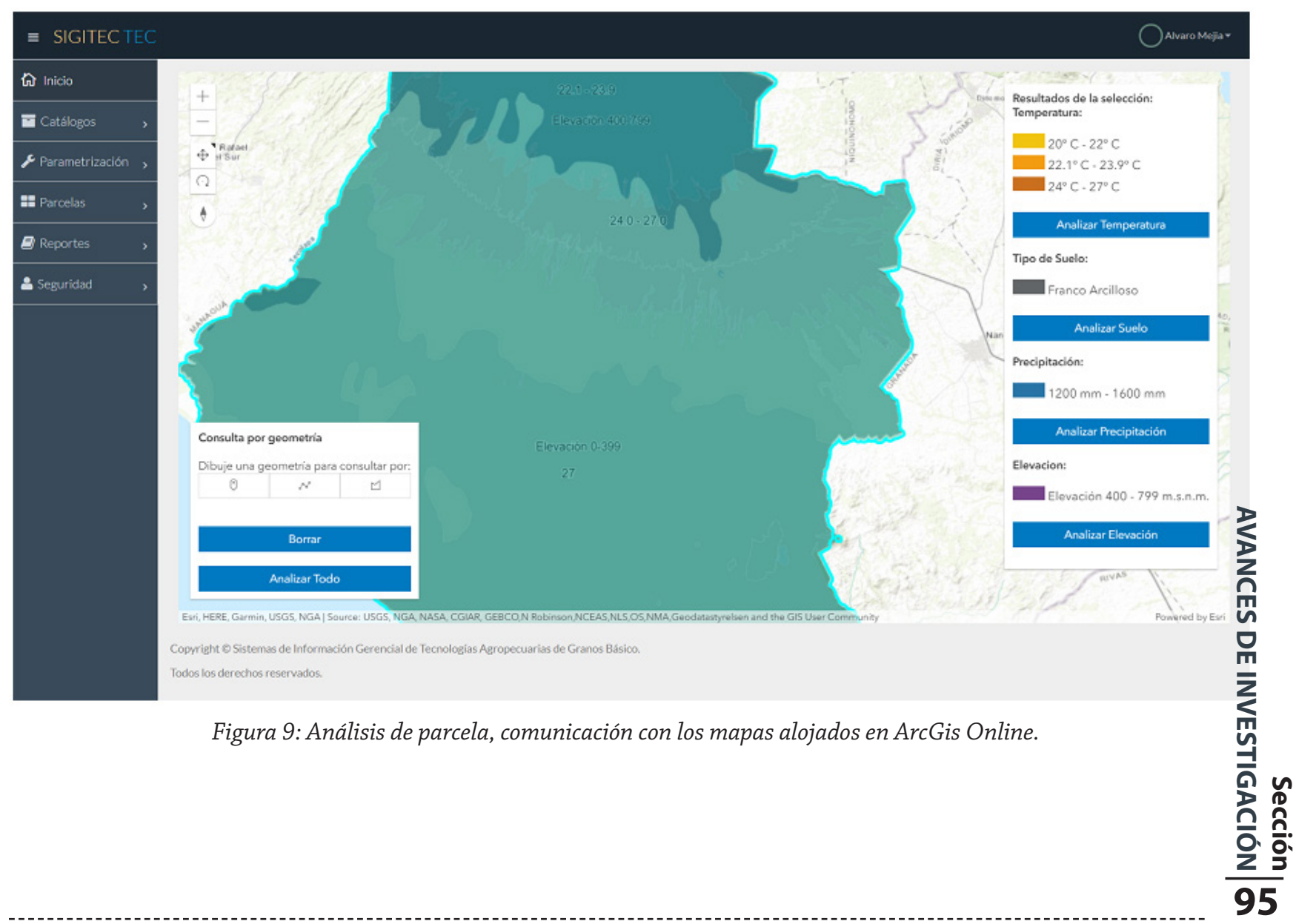




\section{Resultado de variedades}

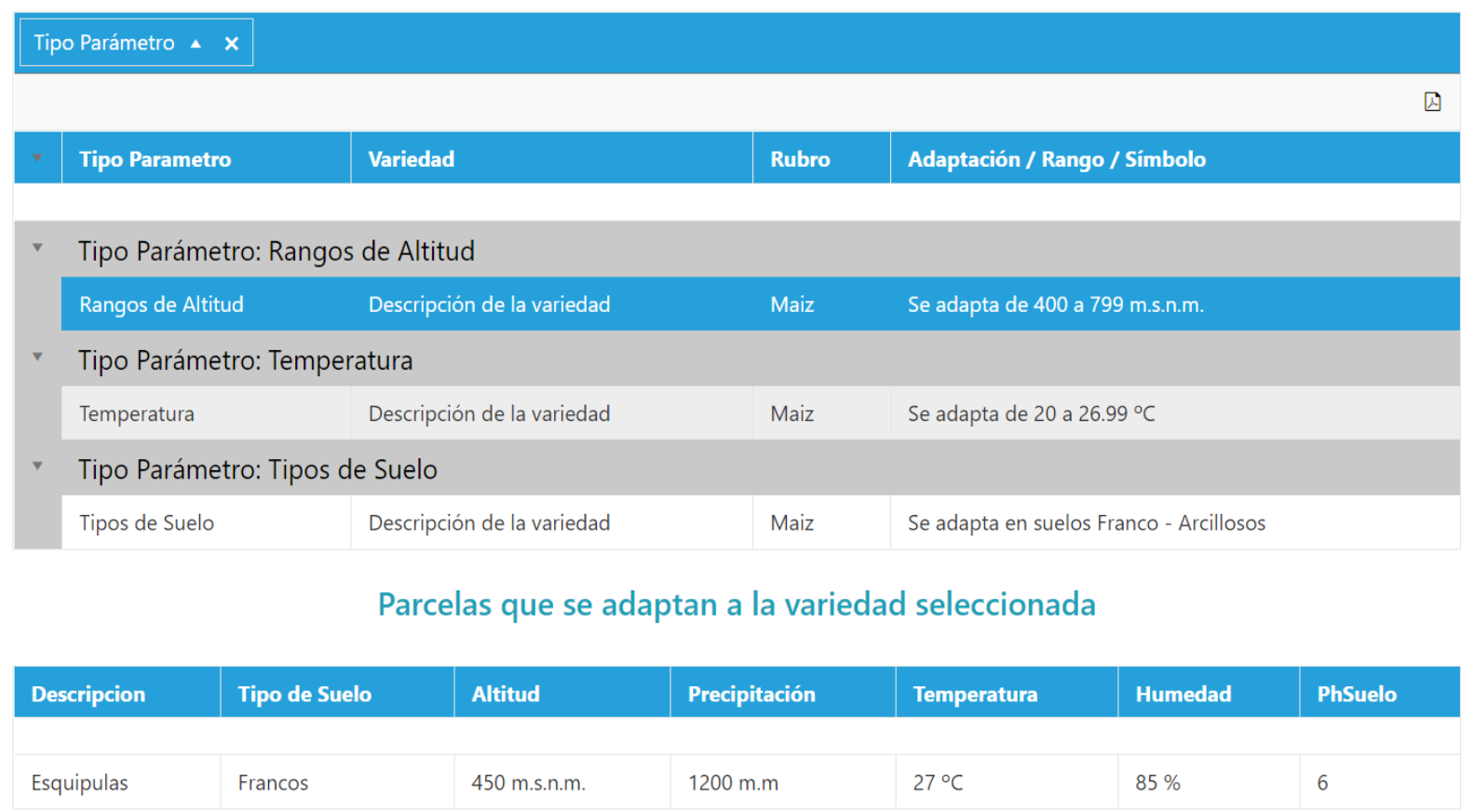

Figura 10: Resultado del Análisis.

\section{DISCUSIÓN}

Para Oliveros, Danyans, \& Mastropietro (2014), las aplicaciones Web requieren nuevos enfoques de la ingeniería de requisitos, que puedan dar una respuesta capaz de ajustarse a una comunidad cambiante, grande y abierta con conocimiento de navegabilidad, respecto a los resultados del capítulo anterior, se tiene como punto de inicio, para el proceso de Desarrollo, la fase de Análisis previa al Diseño y la Implementación, que constituyen en sí mismas actividades investigativas, puesto que con ellas se recolectó, procesó y analizó los datos más relevantes que permitieron el establecimiento de los alcances y limitantes los cuales se llevaron a cabo con: docentes de la Universidad Nacional Agregaría (UNA), técnicos del INTA del Departamento de Carazo y un grupo de 8 productores de la comunidad del Abra que conforman una banco de semilla de arroz en el Municipio de La Conquista, toda esta información se utilizó para la generación de la base de datos.

En este sentido la información necesaria que se recolectó fueron las siguientes:

Con respecto a la información general de cada variedad: Datos de la persona investigadora de la tecnología, Rubro y el nombre de la Variedad, Nombre común o sinónimos de dicha variedad, Instituciones responsables de la selección del material y liberación como tecnología, además del año en que fue liberada, Fotografías de la planta, de la semilla y el perfil de dicha planta (ya sea de Mazorcas, Espigas, Vainas, Granos, Plantío) 
Características Genéticas o genealógicas específicas de: Progenitor de la Variedad y Origen del Progenitor, Características específicas de la genética de la variedad (hibrido doble, triple, etc.), Demás características importantes.

Características Morfológicas de la variedad como: Tamaño de la planta, Altura de inserción de la mazorca, Hábitos de Crecimiento, Color del Grano, Otras características importantes especificados en la boleta de cada variedad.

Características organolépticas: Tiempo de cocción, Calidad de las proteínas (balance de aminoácidos esenciales), Si la variedad es biofortificada.

Disponibilidad de la semilla Genética, básica, registrada, certificada y otra categoría: Cuantificación de semillas de categorías selectas producidas por los INIAS (Institutos de investigación agropecuaria en este caso el INTA) y las empresas privadas o públicas que producen la semilla de la variedad, Descripción de los volúmenes de semillas presentes en el mercado y sus distribuidores.

Características Morfológicas de la variedad como: Tamaño de la planta, Altura de inserción de la mazorca, Hábitos de Crecimiento, Color del Grano, Otras características importantes especificados en la boleta de cada variedad.

Características Agronómicas de la Variedad: Rendimiento potencial, Adaptación a diferentes suelos con limitantes (suelos salinos, suelos ácidos, con mal drenaje, bajos en nitrógeno, etc.), Tolerancia o resistencia a patógenos (hongos, virus, bacterias, micro plasmas, etc.) y a insectos y plagas, Épocas de siembra de preferencias.

Parámetros Biofísicos a los que se adapta la variedad: Rangos de altitud sobre el nivel del mar (m.s.n.m), Tipos de suelos (francos, limosos, arcillosos, arenosos y las derivaciones de cada uno de ellos), Precipitación pluvial (mm de lluvias caídas sobre una zona), Temperaturas (en $\mathrm{C}^{\circ}$ ), Humedad Relativa (\%), pH del suelo.

El sistema permite almacenar: Cantidad de Hectáreas cultivadas de una variedad, Capacidad biofísica para la producción de una variedad, Distribución por zona geográfica en el departamento, Áreas Cultivadas y distribución geográficas en el Departamento de Carazo, Áreas en hectáreas o manzanas de cultivo en el departamento, Un mapa con las zonas donde existen los cultivos de dicha variedad, con la distribución geográfica correspondiente, para esto cada levantamiento de las variedades deberán tener las coordenadas geográficas en el sistema oficial de georreferenciación del país, Estimación de la adopción del material en el departamento, Volumen de producción de la variedad por cada zona, Rendimiento para cada rubro en las zonas cultivadas, Estimación del grado de adopción del grano en el departamento. 
Con lo anteriormente mencionado, se procedió a la definición de la tecnología de software y la metodología a utilizar, y para esto, a como lo menciona Molina R., Zea O., Contento S., \& García Z., (2018), al comparar metodologías como: SOHDM, OOHDM, WSDM, WAE, UWE y IWEB, a partir del análisis de la información y comparación en el marco de desarrollo de su investigación, se obtuvo como resultados que la metodología OOHDM es la que cumple con casi todas las características para el proceso de desarrollo de aplicaciones Web. Para la utilización de esta metodología se utilizó el diagramado UML, con lo cual se representaron las entidades y relaciones e interacciones entre estas.

Para evaluar la factibilidad y viabilidad del desarrollo del software, se tomó en cuenta lo establecido por Oliveros et al., (2014), en el que definen las aplicaciones Web como tecnología que requiere métodos y herramientas especiales para su utilización, además de conocimientos técnicos y científicos para su desarrollo, para esto se analizó la propuesta con base en el conocimiento del lenguaje, la vinculación con C\# y la plataforma ArcGis Online a como se muestra en la Tabla 1.

Con respecto al desarrollo y la tecnología utilizada para la comunicación de la base de datos, se contó con la ventaja de la documentación acerca del servicio de consumo de mapas online que puede hacerse desde C\# a ArcGis Online, para esto se procedió a codificar y consumir el servicio web que permitió esta comunicación entre la base de datos de SQL Server y los Mapas.

En las siguientes figuras se puede apreciar el ciclo de vida de software utilizado y el diagrama general de comunicación. 


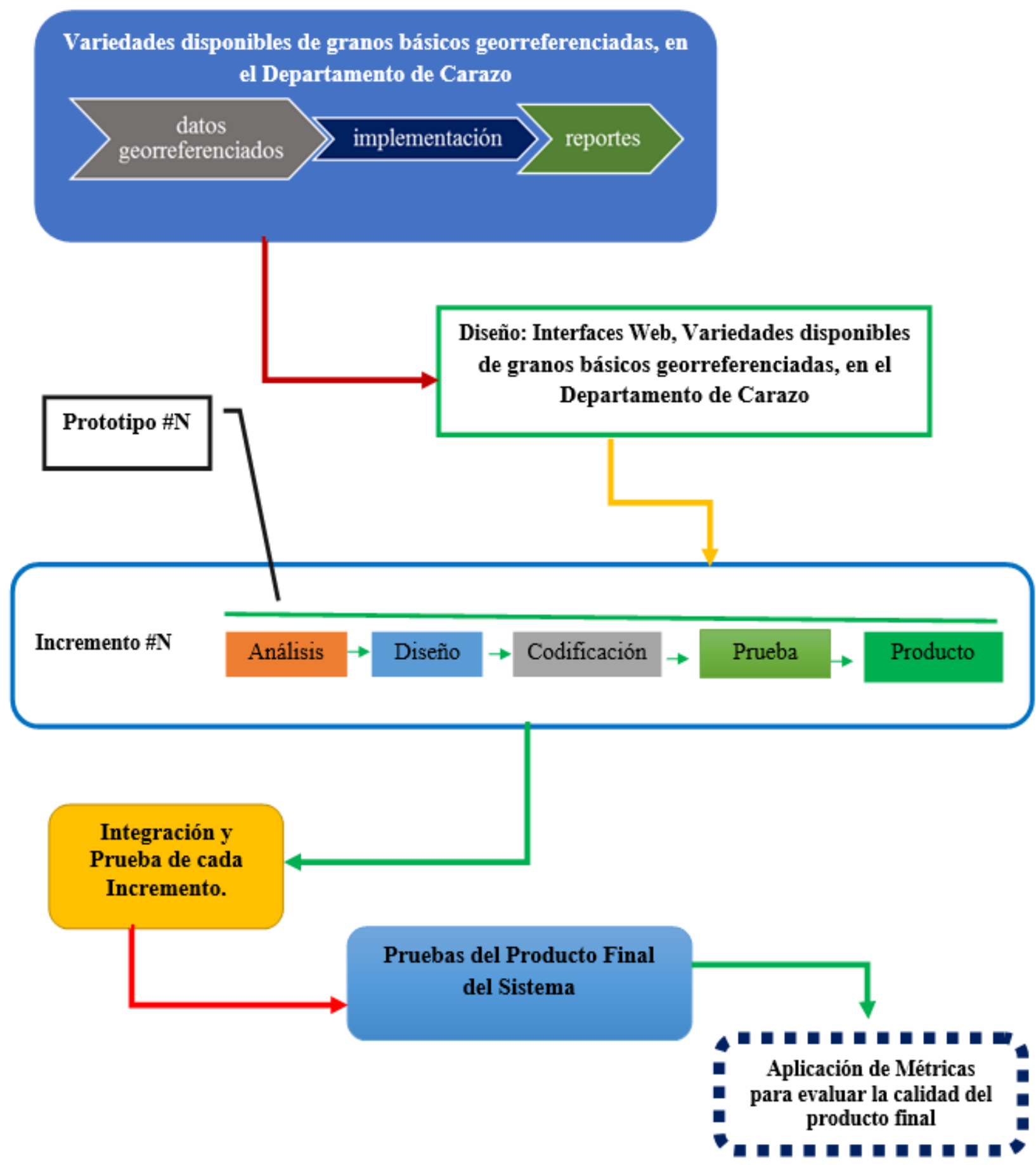

Figura 11: Ciclo de Vida del desarrollo del sistema. 


\section{Dispositivos}

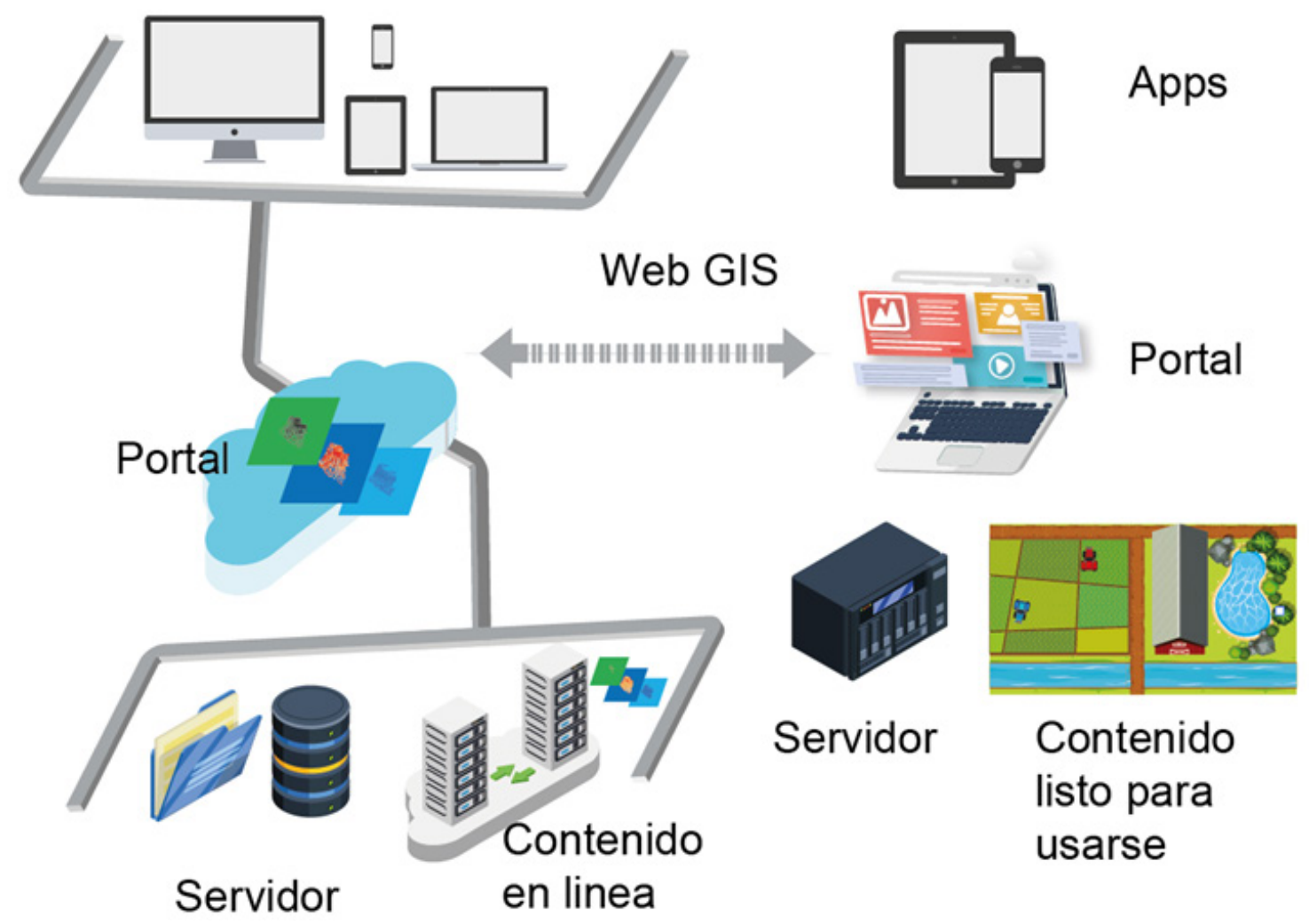

Figura 12: Diagrama de comunicación servidor de sistema y servidor de mapas.

\section{CONCLUSIONES}

Después de haber realizado las entrevistas a los docentes de la UNA, Técnicos del INTACarazo y agricultores, en las cuales se abordó sobre cómo debían estar estructurados los módulos del sistema, entradas y salidas que este debería obtener y mostrar respectivamente. Se obtuvo como principales resultados, la definición de los alcances y limitantes del sistema, lo cual se tomó como punto de partida para iniciar con la definición de los requerimientos del sistema web

En concordancia a lo anteriormente expuesto y siguiendo con la definición de los objetivos específicos planteados, se procedió a la realización del estudio de factibilidad técnica tomando en cuenta las bondades de las tecnologías seleccionada. Esta decisión fue tomada teniendo en cuenta las experiencias en Sistemas de Información Geográfica y el lenguaje de programación seleccionado

Con la información recopilada y la plataforma de desarrollo elegida se continuó con la etapa de análisis y diseño del Sistema de Manejo de tecnologías de granos básicos, para lo cual se procedió a realizar el diagramado utilizando el paradigma UML y la metodología OOHDM (siendo esta la mejor evaluada según Molina Ríos et al., (2018)), para el diseño, en lo relativo a 
lo conceptual, navegacional e interfaces, con la finalidad de crear un producto intuitivo y de gran utilidad.

El uso de la plataforma de ArcGis Online, dio las pautas necesarias para la comunicación entre la base de datos desarrollada en SQLServer 2018ylos mapas temáticos quefueron elaborados para ser consumidos en tiempo real y poder determinar la adaptabilidad de las variedades de granos básicos a condiciones biofísicas establecidas en la ingeniería de requerimientos de la primera etapa.

\section{BIBLIOGRAFÍA}

Angelov, P., Iglesias, J. A., \& Corrales, J. C. (2017). Advances in Information and Communication Technologies for Adapting Agriculture to Climate Change (C. Springer, Ed.). Recuperado de https://doi.org/https:// doi.org/10.1007/978-3-319-70187-5

Banco Interamericano de Desarrollo. (2012). Nicaragua: Estrategia del BID con el país (2012-2017).

Corrales, J. C., Angelov, P., \& Iglesias, J. A. (2018). Advances in Information and Communication Technologies for Adapting Agriculture to Climate Change II. Recuperado de https:// doi.org/https://doi.org/10.1007/978-3030-04447-3

Instituto Nicaraguense de Tecnología Agropecuaria, I. (2015). Catálogo de tecnologías para enfrentar el cambio climático.

Instituto Nicaraguense de Tecnología Agropecuaria, I. (2019). Agricultura Inteligente. Recuperado de http://www. inta.gob.ni/agricultura-inteligente/

MicroSoft. (2019). Entity Framework overview. Recuperado de https://docs.microsoft.com/ en-us/dotnet/framework/data/adonet/ef/ overview
Molina Ríos, J. R., Zea Ordóñez, M. P., Contento Segarra, M. J., \& García Zerda, F. G. (2018). Comparación De Metodologías En Aplicaciones Web. 3C Tecnología_ Glosas de Innovación Aplicadas a La Pyme, 7(1), 1-19. Recuperado de https://doi. org/10.17993/3ctecno.2018.v7n1e25.1-19

Mundial, B. (2019). Nicaragua: panorama general. Retrieved from Nicaragua: panorama general Tomado de: https://www. bancomundial.org/es/country/nicaragua/ overview

Oliveros, A., Danyans, F. J., \& Mastropietro, M. L. (2014). Prácticas de ingeniería de requerimientos en el desarrollo de aplicaciones web. CIBSE 2014: Proceedings of the 17th Ibero-American Conference Software Engineering, 491-505.

Pérez Navarro, A. (2011). Introducción a los sistemas de información geográfica $y$ geotelemática (U. O. de Catalunya, Ed.). Recuperado de http://www. digitaliapublishing.com/a/20241/ introduccion-a-los-sistemas-deinformacion-geografica-y-geotelematica 